\title{
Forest liming durably impact the communities of ectomycorrhizas and fungal epigeous fruiting bodies
}

\author{
François RINEAU ${ }^{1 *}$, Jean-Paul MAURICE ${ }^{2}$, Claude NYS $^{3}$, Hubert VOIRY $^{4}$, Jean GARBAYE ${ }^{1}$ \\ ${ }^{1}$ UMR 1136 INRA/Nancy Université Interactions Arbres/Microorganismes, INRA Nancy, 54280, Champenoux, France \\ ${ }^{2}$ Groupe Mycologique Vosgien, 18 bis, place des Cordeliers, 88300 Neufchâteau, France \\ ${ }^{3}$ Biogéochimie des Écosystèmes Forestiers, INRA Nancy, 54280, Champenoux, France \\ ${ }^{4}$ Office National des Forêts, route de Bussang, 88200 Remiremont, France
}

(Received 5 March 2009; accepted 6 July 2009)

Keywords:

mycorrhiza /

ecosystem management

Mots-clés :

mycorhize /

management des écosystèmes

\begin{abstract}
- Liming is a forestry practice used to counteract forest decline in acidic soils. It consists of direct $\mathrm{Ca}$ and $\mathrm{Mg}$ input to forest soil, which restores tree mineral nutrition, but also modifies microbial communities in soil. The aim of this study was to assess the effects of liming on both belowground (ectomycorrhizal root tips) and aboveground (epigeous sporocarps) fungal communities.

- Results showed that the modification of soil chemical properties $(\mathrm{pH}$, and Ca-Mg contents versus total free $\mathrm{Al}$ and $\mathrm{Fe}$ concentrations) was a stronger factor of ECM community structuring than tree host. The species appearing in limed plots were ubiquist or known as good competitors and replaced acidophilic and stress species.

- At the sporocarp level, tree host was a stronger factor of community structuring than soil chemical properties associated with liming. On the whole, there was a shift in the community composition from a typical acidophilic forest fungal community of medium altitude in the untreated plots to a less typical one, with the reduced dominance of acidophilic fungi while many late-stage forest species appeared.

- We finally suggest a marker species (Russula ochroleuca) to assess both above and belowground effects of liming on ectomycorrhizal communities.
\end{abstract}

Résumé - Le chaulage forestier influence durablement les communautés d'ectomycorrhizes et de carpophores épigés.

- Le chaulage est une pratique forestière utilisée pour restaurer la nutrition minérale des arbres apparaissant sur sol acide. Il consiste en un apport direct de $\mathrm{Ca}$ et $\mathrm{Mg}$ au sol forestier, ce qui restaure la nutrition minérale de l'arbre, mais aussi modifie les communautés microbiennes du sol. Cette étude évalue les effets du chaulage sur les communautés fongiques hypogées (apex ectomycorrhiziens : «ECM ») et épigées (carpophores).

- Les résultats montrent que la modification des des propriétés chimiques du sol ( $\mathrm{pH}$ et concentrations en $\mathrm{Ca}-\mathrm{Mg}$ échangeables versus concentrations en $\mathrm{Al}$ et Fe échangeables) est un facteur de structuration de la communauté d'ECMs plus fort que l'arbre hôte. Les espèces qui sont apparues dans les placeaux chaulés sont ubiquistes ou compétitrices et ont remplacé des espèces acidophiles ou connues pour être associées à des conditions de stress.

- Concernant les carpophores, l'arbre hôte est un facteur de structuration de la communauté plus fort que les propriétés chimiques du sol associées au chaulage. Dans l'ensemble, on a observé une modification de la communauté fongique, passant d'une communauté typique de forêt acide de moyenne altitude dans les placeaux témoins vers une autre moins spécifique, caractérisée par une moindre dominance d'espèces acidophiles et l'apparition de nombreuses espèces de forêt mature.

- Nous suggérons enfin une espèce marqueur (Russula ochroleuca) qui permet d'évaluer facilement les effets du chaulage sur les communautés de champignons mycorrhiziens, aussi bien du point de vue des apex mycorrhiziens que des carpophores.

\footnotetext{
*Corresponding author: francois.rineau@laposte.net
} 


\section{INTRODUCTION}

Symptoms of forest decline due to soil acidification have been reported in many forests of central Europe for the past 20 y (Ulrich, 1983). Spruce needle yellowing and beech defoliation were consequences of cation starvation due to free calcium and magnesium losses in acidic soils with low weathering rates. Liming (i.e. direct input of $\mathrm{Ca}$ and $\mathrm{Mg}$ in forest soils) proves to be efficient to restore tree mineral nutrition, and induces drastic and long-term changes in soil structure and chemical properties (Kreutzer, 1995). Calcareous amendments reduce the acidity of upper soil layers, increase cation exchange capacity and soil base saturation, especially in humus layers (Frank and Stuanes, 2003; Kreutzer, 1995; Renaud et al., 2000), and stimulates soil fauna (particularly earthworms) (Persson, 1988). Liming could also shift the humus type from mor to mull (Kreutzer, 1995).

Liming is now once again a topical subject for forest management as a tool to improve the production of wood biomass in nutrient-poor forest ecosystems. As this forest practice will be applied to large forest areas, ecological consequences of calcareous amendments are thus of critical importance. Renaud et al. (2000) showed increased plant species richness due to the apparition of meso-acidophilic to neutrophilic or nitrophilic species in limed spruce and fir forests. However, the abundance decrease of acidophilic species followed by appearance of widespread neutro- and nitrophilic ones could lead to a shift of patrimonial value of the ecosystem.

Because fungi are strongly influenced by soil organic matter and soil properties, the effect of liming on ectomycorrhizal (ECM) communities has already been surveyed. Liming enhanced sporocarp production of Hygrophorus pustulatus (Agerer et al., 1998) in spruce stands, and of Lycoperdon gemmatum in beech ones (Garbaye et al., 1979). Contrary to that, sporocarps of the acidophilic (but widespread) fungus Russula ochroleuca decreased in abundance in limed spruce stands (Agerer et al., 1998). In terms of ectomycorhizal root tips, very diverse effects of liming were recorded, probably because of site-dependant response to amendment. A promoting effect of liming on ECM root tip abundance was reported in oak plots (Bakker et al., 2000) and in the humic layers of a Norway spruce stand (Nowotny et al., 1998), but the opposite effect was observed in a mixed oak and beech forest (Blaise and Garbaye, 1983). Haug and Feger (1990) showed no significant effect of liming on relative frequency of mycorrhizal and non-mycorrhizal roots. When focusing on the relative abundances of certain ECM species, liming increased the abundance of ECMs of Piceirhiza nigra and Tuber puberulum in spruce forests (Qian et al., 1998). Investigation on the effects of liming on the exploration type of ECMs (according to Agerer, 2001) showed the reduced abundance of ECMs with abundant external mycelium (medium- and long-distance exploration types) in limed plots (Blaise and Garbaye, 1983). Nevertheless, most of the works performed to date, assessed effects of liming on a short time scale (less than $10 \mathrm{y}$ ) and without parallel survey of ECM root tips and the fruiting bodies (FB) of symbiotic and saprophytic fungal species.
Here, we addressed the hypothesis that, as for plants, liming shifts the ECM community structure from acidophilic to a more widespread and neutrophilic type. The aim of this paper is thus to evaluate the mid-term (i.e. $15 \mathrm{y}$ ) impact of liming on the structure of both belowground (mycorrhizal root tips) and aboveground (epigeous sporocarps) fungal communities and to relate it with soil chemical properties.

\section{MATERIAL AND METHODS}

\subsection{Study site}

The experimental site of Humont $\left(48^{\circ} 00^{\prime} 00^{\prime \prime} \mathrm{N}, 6^{\circ} 29^{\prime} 28^{\prime \prime} \mathrm{E}\right.$, Elevation: $570 \mathrm{~m}$, Vosges mountains, North-Eastern France) consists in moderately declining stands of 35-year old Norway spruce (Picea abies) and 60-year-old beech (Fagus sylvatica). The liming treatment was carried out in part of these stands by helicopter in 1991 with $757 \mathrm{~kg} / \mathrm{ha}$ of $\mathrm{CaO}$ and $380 \mathrm{~kg} / \mathrm{ha}$ of $\mathrm{MgO}$, as grounded dolomitic rock, which is a relatively low dose compared to most calcareous amendments in eastern europe. The allocrisol (typic dystrochrept, USDA, 1999) is formed on sandstone. Four blocks were defined, two under beech (B) and two under spruce (S); in each block a couple of plots (limed: L, and untreated: $\mathrm{U}$ ) were defined, resulting in 8 plots of about $20 \mathrm{~m} \times 20 \mathrm{~m}$ each. Fifteen years after the treatment, liming had resulted in a shift of the humus type from moder to oligomull, restored tree health, mineral nutrition and vegetation diversity and strongly increased earthworm colonisation (Renaud et al., 2000).

\subsection{Soil analyses}

The topsoil layers of five soil cores ( $8 \mathrm{~cm}$ in diameter, $20 \mathrm{~cm}$ deep) were taken in each plot in October 2006. The five cores were then pooled per plot, air-dried at ambient temperature during one week and sieved at $2 \mathrm{~mm}$. We then measured, for each composite sample, the total contents of $\mathrm{N}$, organic matter, $\mathrm{pH}$, and concentrations of exchangeable $\mathrm{Al}^{3+}, \mathrm{Mg}^{2+}, \mathrm{Fe}^{3+}, \mathrm{Ca}^{2+}, \mathrm{Mn}^{2+}, \mathrm{K}^{+}$and $\mathrm{Na}^{+}$(Thomas, 1982). Soil phosphorus contents were estimated using the Duchaufour method, consisting in a double acid and basic extraction, which solubilized both organic and mineral P components (Duchaufour and Bonneau, 1959).

\subsection{Estimation of the ECM root tip community structure}

Soil core sampling was carried out on October 2006 (before leaf fall, date 1), May 2007 (after bud break, date 2) and October 2007 (before leaf fall, date 3). At each date, ECM sampling was done in one of the two blocks for beech and spruce, in order to measure ECM community structure at least once for each treatment. Three soil cores ( $8 \mathrm{~cm}$ diameter, $15 \mathrm{~cm}$ deep, $750 \mathrm{~cm}^{3}$ ) were randomly collected in each sampled plot for date 1 . Twenty soil cores $(4 \mathrm{~cm}$ diameter, $18 \mathrm{~cm}$ deep, $225 \mathrm{~cm}^{3}$ ) were randomly collected in each sampled plot for date 2 . Twenty soil cores of the same size were collected in each sampled plot for date 3 in a $5 \times 4$ grid (distance between two soil cores: $2.5 \mathrm{~m}$ ). As the aim of this study was to estimate the effects of liming on ECM communities, the effect of season (spring vs. autumn) on community structure was not taken into account here, 
and the spring 2007 sampling was considered as an independent time replicate. Considering that the ECM community structure is variable at a very small space scale (Dahlberg, 2001), three soil cores a few meters apart and thoroughly investigated took into account as much as variability in the ECM community than 20 smaller soil cores. The community structure obtained with the three sampling schemes was thus comparable.

Ectomycorrhizal morphotypes were then identified according to Agerer (1987-98). For each collected morphotype, seven tips were frozen at $-80{ }^{\circ} \mathrm{C}$ and used, when possible, for extending the morphotype identification by DNA sequencing of the ITS region (Gardes and Bruns, 1993). Sequences were aligned using ITS sequences available in the NCBI (http://www.ncbi.nlm.nih.gov/) and UNITE (http:// unite.ut.ee/) databases. The morphological description and the results of DNA identification of all morphotypes are given in the supplementary Table I.

Ectomycorrhizal tips of each species were exhaustively counted in soil cores at date 1 . For the date 2 samples, roots were washed and homogeneously spread in a large Petri dish $(20 \mathrm{~cm}$ diameter) containing tap water. Then ECM tips of each species were exhaustively counted in a $1 / 8$ sector of the dish. For the sample date 3 , roots were washed, homogeneously spread in the same type of Petri dish, and cut in small pieces ( $1 \mathrm{~cm}$ long). Root pieces were then randomly picked and ECMs counted until we reached 100 tips (Garbaye, 1990). For each date, values of abundance were then transformed into relative abundances by dividing the total number of ECM root tips belonging to a given species by the total number of root tips found in the plot. We then calculated the mean relative abundance of each species at the three sampling dates. We then compared the mean relative abundances of all species during the whole sampling campaign.

\subsection{Counting of sporocarps}

Only conspicuous, epigeous sporocarps were counted; hypogeous and hidden resupinate ones were ignored. At each date, fungal sporocarps were identified and counted in at least one block under spruce or beech, in order to measure sporocarp community structure at least one time for each block. Fruiting bodies were absent in May 2007, so the sampling date 2 was postponed to the beginning of July 2007 for sporocarp counting. In addition, we assessed the presence/absence of saprophytic species in each block. Areas from $1600 \mathrm{~m}^{2}$ to $3200 \mathrm{~m}^{2}$, equally distributed between treatments, were surveyed by the same two people, for up to $1 \mathrm{~h}$ per block, at each sampling date.

\subsection{Statistics}

The Shannon diversity index $\left(H^{\prime}\right)$ was calculated as: $H^{\prime}=-\sum((N i / N) \times \log 2(N i / N))$, and the Simpson's equitability index was calculated as: $D=\sum N i(N i-1) /(N(N-1))$, where $\mathrm{Ni}$ was the abundance of the species $i$ and $N$ the total number of individuals. Diversity indices (Shannon index, Simpson index) were then compared between limed and untreated plots for each tree host using a student $t$ test.

Fungal communities structures in each treatment were compared using canonical correspondence analysis (CCA) based on soil analyses with relative abundances of the species (ECM root tips and sporocarps of ECM fungi) and presence/absence data (saprophytic species). Statistical calculations and representations were done using the R software (http://www.r-project.org) (Ihaka and Gentleman, 1996).

\section{RESULTS}

\subsection{Description of the ECM fungal communities}

Throughout the whole duration of the study, 40 morphotypes of ECM root tips were observed, and sporocarps of 52 species of ECM fungi and of 70 species of saprophytic fungi were found.

Concerning the ECM community, the most abundant morphotype was Cenococcum geophilum, always more abundant in the untreated plots ( 34 to $69 \%$ vs. 13 to $46 \%$ in the limed one, as seen in Tab. I). In the same way, Russula ochroleuca ECMs were almost absent in the limed plots $(0.5 \%$ abundance in one spruce limed plot) but relatively abundant and present in all the untreated ones ( 1 to $11 \%$ ). The limed plots were dominated by Clavulina cristata ECMs (except for SL$\mathrm{B}$ one), under beech as well as under spruce, except in one replicate of the spruce limed plots. Lactarius subdulcis and Tomentella sublilacina were also found as codominant species in the beech limed plots. Ectomycorrhizae of Xerocomus pruinatus were found at low abundances but in all plots, whatever in limed or untreated ones. The ECM fungus Laccaria amethystina was not frequent but locally abundant in some beech plots.

Concerning sporocarps, Russula ochroleuca, Laccaria amethystina and Inocybe napipes were found in all the treatments, and Russula ochroleuca was the only species found in all the blocks (Tab. I). The fungal species Amanita citrina was the only species to be present only in untreated plots, whatever the tree host, and absent in limed ones. In the spruce untreated plot, the sporocarp community was dominated by Russula ochroleuca (51\% of relative abundance, in mean between the two plots) and to a lesser extent Hygrophorus olivaceoalbus (24\%). In spruce limed plots, the community was dominated by Clavulina cristata (46\% in mean, but especially dominant in one replicate with $91 \%$ abundance), Russula ochroleuca (19\%), and Lactarius tabidus (12\%), but with large differencies between the two plots. Amanita rubescens and many other rare species $(<3 \%$ relative abundance: Amanitopsis submembranacea, Cortinarius evernius, Elaphomyces muricatus, Lactarius helvus, Russula puellaris, Russula turci) appeared in these limed spruce plots, while the abundance of the fungus Xerocomus was slightly repressed (from 2 to $5 \%$ in untreated plots vs. 1 to $2 \%$ in limed ones).

The consequences of liming under beech were less obvious because total abundances were not so high. In the untreated plots, the community was dominated by Russula ochroleuca (20\% in mean between the two plots), Russula fageticola (17\%) and Cortinarius delibutus (10\%). In beech limed plots, the two latter species were absent and the community was dominated by Laccaria laccata $(32 \%)$, Russula ochroleuca (13\%) and Russula cyanoxantha (13\%). We observed the apparition of Clavulina cristata and many Amanita and Russula species (Amanita spissa, Amanita battarae, Amanita eliae, Russula heterophylla, Russula lilacea, Russula parazurea); moreover, the relative abundance of other Amanita spp. (Amanitopsis submembranacea) and Russula spp. (Russula brunneoviolacea, Russula cyanoxantha) was 
Table I. Relative abundance of ECM fungi in each of the 8 plots. S: spruce, B: Beech, U: untreated, L: limed, A: replicate A, B: replicate B. The intensity of the grey shading increases with higher values. The abbreviation name used for each species in Figures 1 and 2 is given in brackets in the first column.

\begin{tabular}{|c|c|c|c|c|c|c|c|c|c|c|c|c|c|c|c|c|}
\hline \multirow{4}{*}{$\begin{array}{l}\text { Tree host } \\
\text { Treatment } \\
\text { Replicate }\end{array}$} & \multicolumn{8}{|c|}{ ECM root tips } & \multicolumn{8}{|c|}{ Sporocarps } \\
\hline & \multicolumn{4}{|c|}{ Beech } & \multicolumn{4}{|c|}{ Spruce } & \multicolumn{4}{|c|}{ Beech } & \multicolumn{4}{|c|}{ Spruce } \\
\hline & \multicolumn{2}{|c|}{ Limed } & \multicolumn{2}{|c|}{ Untreated } & \multicolumn{2}{|c|}{ Limed } & \multicolumn{2}{|c|}{ Untreated } & & med & Untre & treated & Lin & ned & Untre & eated \\
\hline & A & $\mathrm{B}$ & A & $\mathrm{B}$ & A & $\mathrm{B}$ & A & $\mathrm{B}$ & A & $\mathrm{B}$ & A & $\mathrm{B}$ & A & $\mathrm{B}$ & A & $\mathrm{B}$ \\
\hline$\overline{\text { Amanita battarrae (Amba) }}$ & & & & & & & & & $6.45 \%$ & & & & & & & \\
\hline Amanita citrina (Amci) & & & & & & & & & & & $2.67 \%$ & & & & $1.05 \%$ & \\
\hline Amanita crocea $($ Amcr $)$ & & & & & & & & & $3.23 \%$ & & & & & & & \\
\hline Amanita eliae (Amel) & & & & & & & & & $16.12 \%$ & & & & & & & \\
\hline Amanita rubescens (Amru) & $5.58 \%$ & $0.36 \%$ & $0.08 \%$ & $1.49 \%$ & & & & & & & $1.33 \%$ & & & $3.60 \%$ & & \\
\hline Amanita sp. (Amsp) & & & & & $0.35 \%$ & & & $0.49 \%$ & & & & & & & & \\
\hline Amanita spissa (Amspi) & & & & & & & & & & $11.11 \%$ & & & $0.46 \%$ & & & \\
\hline Amanitopsis & & & & & & & & & $3.23 \%$ & & $1.33 \%$ & & & $0.72 \%$ & & \\
\hline submembranacea (Amsub) & & & & & & & & & & & & & & & & \\
\hline Boletus edulis (Boel) & & & & & & & & & & & $1.33 \%$ & & & & & \\
\hline Cantharella sp. (Casp) & & & $0.04 \%$ & & & & & & & & & & & & & \\
\hline Cantharellus cibarius (Caci) & & & & & & & & & & & & & & & & $0.64 \%$ \\
\hline Cenococcum geophilum (Cege) & $13.23 \%$ & $26.03 \%$ & $71.73 \%$ & $34.07 \%$ & $15.39 \%$ & $45.75 \%$ & $50.99 \%$ & $69.59 \%$ & & & & & & & & \\
\hline Clavulina cristata (Clcr) & $18.68 \%$ & $17.97 \%$ & $5.60 \%$ & $8.43 \%$ & $54.56 \%$ & $3.28 \%$ & $1.38 \%$ & & $3.23 \%$ & & & & $91.30 \%$ & $0.72 \%$ & $0.26 \%$ & \\
\hline Cortinarius acutus (Coac) & & & & & & & & & & & & & $0.46 \%$ & & $0.79 \%$ & \\
\hline Cortinarius anomalus (Coan) & & $0.63 \%$ & $0.80 \%$ & & & & $0.01 \%$ & $0.24 \%$ & & & & & & & & \\
\hline Cortinarius argentatus (Coar) & & & & & & & & & & & $1.33 \%$ & & & & & \\
\hline Cortinarius delibutus (Code) & & & & & & & & & & & $9.33 \%$ & $611.11 \%$ & & & & \\
\hline Cortinarius evernius (Coer) & & & & & & & & & & & & & & $0.72 \%$ & & \\
\hline Cortinarius lebretonii (Coer) & & & & & & & & & & & & $11.11 \%$ & $0.46 \%$ & & $1.31 \%$ & \\
\hline Cortinarius sp. (Cosp) & & & $0.14 \%$ & $7.11 \%$ & $0.66 \%$ & & $0.25 \%$ & & & & & & & & & \\
\hline Dermocybe sp. (Desp) & & $0.24 \%$ & $8.21 \%$ & $1.21 \%$ & & & & & & & & & & & & \\
\hline Elaphomyces muricatus (Elmu) & & & & & & & & & & & & & & $2.16 \%$ & & \\
\hline Gomphidius glutinosus (Gogl) & & & & & & & & & & & & & & & & $2.55 \%$ \\
\hline Hygrophorus olivaceoalbus (Hyol) & & & $3.52 \%$ & & $0.02 \%$ & $14.91 \%$ & $3.18 \%$ & & & & & & $1.37 \%$ & $15.11 \% 3$ & $32.29 \% 1$ & $15.92 \%$ \\
\hline Hygrophorus pustulatus (Hypu) & & & & & & & & & & & & & & $5.76 \%$ & & $11.46 \%$ \\
\hline Inocybe asterospora (Inas) & & & & & & & & & & & & $11.11 \%$ & & & & \\
\hline Inocybe lanuginosa (Inla) & & & & & & & & & & & & & & & & $0.64 \%$ \\
\hline Inocybe napipes (Inna) & & & & & & & & & $3.23 \%$ & & & $11.11 \%$ & $0.46 \%$ & $0.72 \%$ & $0.26 \%$ & \\
\hline Inocybe petiginosa (Inpe) & & & & & & & & & $3.23 \%$ & & & & & & & \\
\hline Laccaria amethystina (Laam) & $18.06 \%$ & & & $12.02 \%$ & & & & & $6.45 \%$ & & $1.33 \%$ & $11.11 \%$ & & $6.47 \%$ & $0.26 \%$ & \\
\hline Laccaria laccata (Lala) & & & & & & & & & $9.68 \%$ & $55.56 \%$ & $1.33 \%$ & $11.11 \%$ & & & & \\
\hline Lactarius camphoratus (Laca) & & & & & & & & & & & $2.67 \%$ & & & & $1.31 \%$ & $14.01 \%$ \\
\hline Lactarius helvus (Lahe) & & & & & & & & & & & & & & $2.88 \%$ & & \\
\hline Lactarius sp. (Lasp) & & & $0.96 \%$ & & & & & & & & & & & & & \\
\hline Lactarius sp.2 (Lasp2) & & & $1.19 \%$ & & & & & & & & & & & & & \\
\hline Lactarius sp.3 (Lasp3) & & & & & $1.99 \%$ & & & & & & & & & & & \\
\hline Lactarius sp.6 (Lasp6) & & $9.67 \%$ & & & & & & & & & & & & & & \\
\hline Lactarius subdulcis (Lasu) & $11.60 \%$ & $21.05 \%$ & $1.45 \%$ & $3.20 \%$ & & & & & $3.23 \%$ & & $2.67 \%$ & $11.11 \%$ & & & & \\
\hline Lactarius tabidus (Lata) & & & & & $0.87 \%$ & $4.68 \%$ & $2.89 \%$ & $1.12 \%$ & & & $2.67 \%$ & & $0.46 \%$ & $23.74 \%$ & $2.10 \%$ & \\
\hline Paxillus sp. (Pasp) & & & & $1.87 \%$ & $0.83 \%$ & & $3.85 \%$ & $0.39 \%$ & & & & & & & & \\
\hline Piceirhiza sp. (Pisp) & & & & & $2.33 \%$ & & & $1.20 \%$ & & & & & & & & \\
\hline Russula aeruginosa (Ruae) & & & & & & & & & & & & & & & & $2.55 \%$ \\
\hline Russula brunneoviolacea (Rubr) & & & & & & & & & $3.23 \%$ & & $1.33 \%$ & & & & & \\
\hline Russula cyanoxantha (Rucy) & $1.32 \%$ & & & & & & & & $25.79 \%$ & & $1.33 \%$ & & & & $0.52 \%$ & \\
\hline Russula cyanoxantha & & & & & & & & & & & $1.33 \%$ & & & & & \\
\hline var. pelteraui (Rucyp) & & & & & & & & & & & & & & & & \\
\hline Russula densifolia (Rude) & & & & & & & & & & & & & & & $0.26 \%$ & \\
\hline Russula fageticola (Rufa) & & & & & & & & & & & $33.34 \%$ & & & & & \\
\hline Russula heterophylla (Ruhe) & & & & & & & & & $3.23 \%$ & & & & & & & \\
\hline Russula ionochlora (Ruio) & & & & & & & & & & & & & & & $0.79 \%$ & \\
\hline Russula lilacea (Ruli) & & & & & & & & & $3.23 \%$ & & & & & & & \\
\hline Russula nigricans (Runi) & & & & $2.81 \%$ & & & & & & & & $11.11 \%$ & & & & \\
\hline Russula ochroleuca (Ruoc) & & & $2.32 \%$ & $1.60 \%$ & $0.54 \%$ & & $13.00 \%$ & $4.81 \%$ & $3.23 \%$ & $22.22 \%$ & $28.01 \%$ & $611.11 \%$ & $1.83 \%$ & $35.25 \%$ & $55.40 \%$ & $46.50 \%$ \\
\hline Russula parazurea (Rupa) & & & & & & & & & & $11.11 \%$ & & & & & $0.26 \%$ & \\
\hline
\end{tabular}


Table I. Suite.

\begin{tabular}{|c|c|c|c|c|c|c|c|c|c|c|c|c|c|c|c|c|}
\hline \multirow{4}{*}{$\begin{array}{l}\text { Tree host } \\
\text { Treatment } \\
\text { Replicate }\end{array}$} & \multicolumn{8}{|c|}{ ECM root tips } & \multicolumn{8}{|c|}{ Sporocarps } \\
\hline & \multicolumn{4}{|c|}{ Beech } & \multicolumn{4}{|c|}{ Spruce } & \multicolumn{4}{|c|}{ Beech } & \multicolumn{4}{|c|}{ Spruce } \\
\hline & \multicolumn{2}{|c|}{ Limed } & \multicolumn{2}{|c|}{ Untreated } & \multicolumn{2}{|c|}{ Limed } & \multicolumn{2}{|c|}{ Untreated } & \multicolumn{2}{|c|}{ Limed } & \multicolumn{2}{|c|}{ Untreated } & \multicolumn{2}{|c|}{ Limed } & \multicolumn{2}{|c|}{ Untreated } \\
\hline & $\mathrm{A}$ & $\mathrm{B}$ & A & $\mathrm{B}$ & A & $\mathrm{B}$ & A & $\mathrm{B}$ & A & $\mathrm{B}$ & A & $\mathrm{B}$ & $\mathrm{A}$ & $\mathrm{B}$ & $\mathrm{A}$ & $\mathrm{B}$ \\
\hline$\overline{\text { Russula puellaris (Rupu) }}$ & & & & & & & & & & & & & & $0.72 \%$ & & \\
\hline Russula risigalina (Ruri) & & & & & & & & & $3.23 \%$ & & & & & & & \\
\hline Russula sp. 2 (Rusp2) & & & & & & & $0.21 \%$ & & & & & & & & & \\
\hline Russula turci (Rutu) & & & & & & & & & & & & & $0.46 \%$ & & & \\
\hline Russula vesca (Ruve) & & & & & & & & & & & & & $0.91 \%$ & & & $0.64 \%$ \\
\hline Russula violeipes (Ruvi) & & & & & & & & & & & & & & & $0.26 \%$ & \\
\hline Sebacina epigaea (Seep) & $1.50 \%$ & & & $0.88 \%$ & & & & & & & & & & & & \\
\hline Tomentella sp. (Tosp) & $11.72 \%$ & & & $7.00 \%$ & & & & & & & & & & & & \\
\hline Tomentella sp. 2 (Tosp 2$)$ & & & & $1.98 \%$ & & & & $5.54 \%$ & & & & & & & & \\
\hline Tomentella sublilacina (Tosu) & $14.48 \%$ & $15.56 \%$ & $60.05 \%$ & $7.94 \%$ & $4.70 \%$ & & $1.00 \%$ & & & & & & & & & \\
\hline Tricholoma saponaceum $(\operatorname{Trs} a)$ & & & & & & & & & & & $1.33 \%$ & & & & & \\
\hline Tricholoma sp. (Trsp) & & & & & & $14.26 \%$ & & & & & & & & & & \\
\hline Tylopilus felleus (Tyfe) & & & & & & & & & & & & & & & $0.26 \%$ & \\
\hline$U E C M$ sp.l (UE1) & & & & & $6.99 \%$ & $6.35 \%$ & $12.79 \%$ & $67.11 \%$ & & & & & & & & \\
\hline UECM sp.10 (UE10) & & & & & & & & & & & & & & & & \\
\hline$U E C M$ sp.11 (UE11) & & & & & & $5.27 \%$ & & & & & & & & & & \\
\hline UECM sp.12 (UE12) & & & & & & $0.27 \%$ & & & & & & & & & & \\
\hline UECM sp.13 (UE13) & & & & & & $0.81 \%$ & & & & & & & & & & \\
\hline$U E C M$ sp.14 (UE14) & & & & & & $0.16 \%$ & & & & & & & & & & \\
\hline UECM sp.15 (UE15) & & $1.81 \%$ & & & & & & & & & & & & & & \\
\hline$U E C M$ sp.2(UE2) & & & & & $2.71 \%$ & & $0.85 \%$ & $6.84 \%$ & & & & & & & & \\
\hline UECM sp.5(UE5) & & & $0.15 \%$ & & & & & & & & & & & & & \\
\hline UECM sp.6(UE6) & & & & & & & $0.90 \%$ & & & & & & & & & \\
\hline UECM sp.7(UE 7) & & & $2.73 \%$ & & & & & & & & & & & & & \\
\hline UECM sp.8(UE8) & & & & & & & $1.12 \%$ & & & & & & & & & \\
\hline UECM sp.9(UE9) & & & & & & & $0.28 \%$ & & & & & & & & & \\
\hline Xerocomus badius (Xeba) & & & & & & & & & & & & & $1.83 \%$ & $1.44 \%$ & $2.10 \%$ & $5.10 \%$ \\
\hline Xerocomus chrysenteron $($ Xech $)$ & & & & & & & & & & & $5.33 \%$ & & & & & \\
\hline Xerocomus ferrugineus (Xefe) & & & & & & & & & & & & & & & $0.26 \%$ & \\
\hline Xerocomus pruinatus (Xepr) & $3.82 \%$ & $6.67 \%$ & $1.01 \%$ & $8.38 \%$ & $8.07 \%$ & $4.25 \%$ & $7.29 \%$ & $2.67 \%$ & & & & & & & $0.26 \%$ & \\
\hline Diversity (Shannon index) & 1.82 & 2.08 & 1.19 & 2.20 & 1.58 & 1.70 & 1.68 & 1.19 & 1.15 & 2.44 & 2.06 & 2.20 & 0.45 & 1.88 & 1.18 & 1.61 \\
\hline Equitability (Simpson index) & 0.82 & 0.86 & 0.47 & 0.84 & 0.66 & 0.74 & 0.70 & 0.50 & 0.62 & 0.88 & 0.79 & 0.89 & 0.16 & 0.79 & 0.58 & 0.72 \\
\hline
\end{tabular}

promoted by liming. Oppositely, the abundance of Lactarius subdulcis and Inocybe napipes was higher in the untreated plots, while many Cortinarius spp. (Cortinarius delibutus, Cortinarius lebretonii, Cortinarius argentatus) were absent in the limed plots. There was no significant effect of liming on ECM or FB diversity (Shannon index) or equitability (Simpson index) (Tab. I).

Clavulina cristata was a dominant species of both below(ECM root tips) and aboveground (sporocarps) communities, showing its high fructification rate, contrary to Lactarius subdulcis, dominant in terms of ECM root tips but without any fructification in the limed beech plots.

\subsection{Canonical analysis-ECM root tips}

Results of a CCA based on relative abundances of ECM root tips showed a strong opposition between limed and untreated plots (Fig. 1). The first canonical component (33\% of the total variance, $x$ axis) strongly opposed exchangeable $\mathrm{Mn}^{2+}, \mathrm{Ca}^{2+}$ and $\mathrm{Mg}^{2+}$ contensts, and $\mathrm{pH}$, to $\mathrm{Al}^{3+}, \mathrm{N}, \mathrm{C}$ and
Table II. Chemical properties of the topsoil in the 8 sampling plots. Concentrations in $\mathrm{C}, \mathrm{N}$ and available $\mathrm{P}$ are given in $\mathrm{g} \mathrm{kg}^{-1}$. Concentrations in exchangeable $\mathrm{H}^{+}, \mathrm{Al}^{3+}, \mathrm{Ca}^{2+}, \mathrm{Fe}^{3+}, \mathrm{Mg}^{2+}, \mathrm{Mn}^{2+}, \mathrm{K}^{+}$and $\mathrm{Na}^{+}$are given in cmol $+\mathrm{kg}^{-1}$. The available $\mathrm{P}$ has been measured using the method of Duchaufour and Bonneau (1959).

\begin{tabular}{lcccccccc}
\hline Code & SU-A & SU-B & SL-A & SL-B & BU-AB & U-B & BL-A & BL-B \\
\hline $\mathrm{N}$ & 4.89 & 3.80 & 4.83 & 4.56 & 3.86 & 4.28 & 4.17 & 3.07 \\
$\mathrm{C} / \mathrm{N}$ & 21.10 & 20.80 & 19.90 & 21.00 & 16.40 & 15.80 & 18.70 & 17.50 \\
$\mathrm{C}$ & 103.00 & 79.10 & 95.90 & 96.00 & 63.40 & 67.60 & 78.10 & 53.70 \\
$\mathrm{pH}$ & 4.24 & 4.01 & 4.27 & 4.38 & 4.16 & 4.02 & 4.60 & 4.53 \\
$\mathrm{H}+$ & 1.44 & 1.16 & 1.00 & 0.76 & 0.76 & 0.84 & 0.76 & 0.80 \\
$\mathrm{Al}+$ & 8.10 & 8.91 & 6.74 & 8.39 & 6.95 & 8.20 & 4.86 & 5.22 \\
$\mathrm{Ca} 2+$ & 0.80 & 0.37 & 0.93 & 0.82 & 0.15 & 0.20 & 3.57 & 1.57 \\
$\mathrm{Fe} 3+$ & 0.14 & 0.22 & 0.10 & 0.07 & 0.09 & 0.11 & 0.02 & 0.02 \\
$\mathrm{Mg} 2+$ & 0.44 & 0.25 & 0.44 & 0.39 & 0.24 & 0.22 & 0.96 & 0.47 \\
$\mathrm{Mn} 2+$ & 0.18 & 0.10 & 0.24 & 0.05 & 0.06 & 0.14 & 0.44 & 0.43 \\
$\mathrm{~K}+$ & 0.41 & 0.29 & 0.39 & 0.27 & 0.30 & 0.35 & 0.41 & 0.29 \\
$\mathrm{Na}+$ & 0.05 & 0.04 & 0.04 & 0.04 & 0.04 & 0.03 & 0.05 & 0.03 \\
$\mathrm{P} 2 \mathrm{O} 5$ & 0.17 & 0.17 & 0.19 & 0.22 & 0.27 & 0.23 & 0.19 & 0.20 \\
\hline
\end{tabular}


Table III. Distribution and code name (for Fig. 3) of the 70 species of saprophytic fungi found in the study.

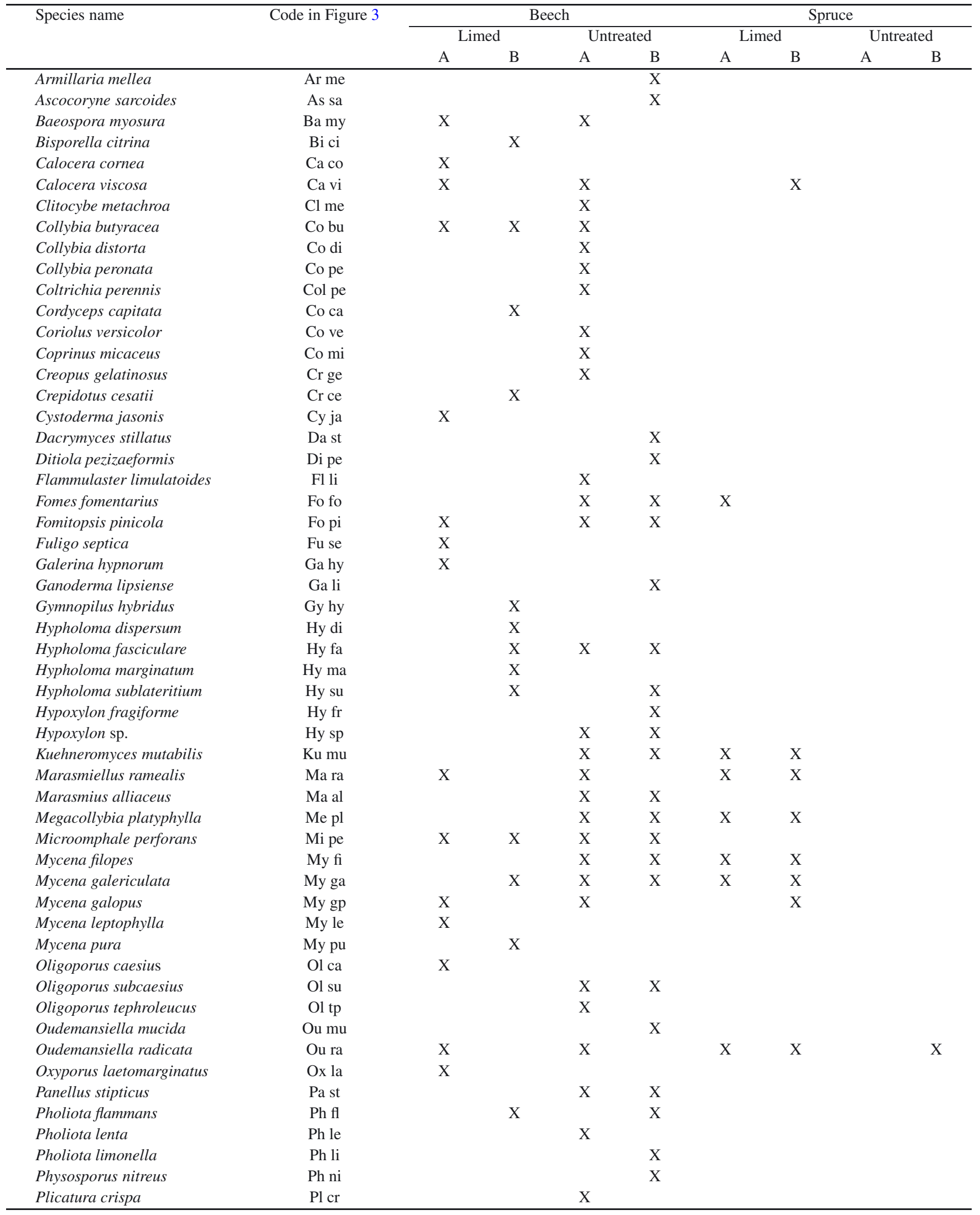


Table III. Suite.

\begin{tabular}{|c|c|c|c|c|c|c|c|c|c|}
\hline \multirow[t]{2}{*}{ Species name } & \multirow[t]{2}{*}{ Code in Figure 3} & \multicolumn{4}{|c|}{ Beech } & \multicolumn{4}{|c|}{ Spruce } \\
\hline & & \multicolumn{2}{|c|}{ Limed } & \multicolumn{2}{|c|}{ Untreated } & \multicolumn{2}{|c|}{ Limed } & \multicolumn{2}{|c|}{ Untreated } \\
\hline Plicaturopsis faginea & $\mathrm{Pl} \mathrm{fa}$ & & & & $\mathrm{X}$ & & & & \\
\hline Rickenella fibula & Ri fi & & & & $\mathrm{X}$ & & & & \\
\hline Schizopora paradoxa & Sc pa & & & & $\mathrm{X}$ & & & & \\
\hline Stereum hirsutum & St hi & & & $\mathrm{X}$ & $\mathrm{X}$ & $\mathrm{X}$ & $X$ & & \\
\hline Trametes gibbosa & Tr gi & & & & $\mathrm{X}$ & & & & \\
\hline Trametes versicolor & Tr ve & & & $\mathrm{X}$ & & $\mathrm{X}$ & $\mathrm{X}$ & & \\
\hline Tremella foliacea & $\operatorname{Tr}$ fo & & $\mathrm{X}$ & & & & & & \\
\hline Tyromyces stipticus & Ty st & & $\mathrm{X}$ & & & & & & \\
\hline Ustulina deusta & Us de & & & & $\mathrm{X}$ & & & & \\
\hline
\end{tabular}

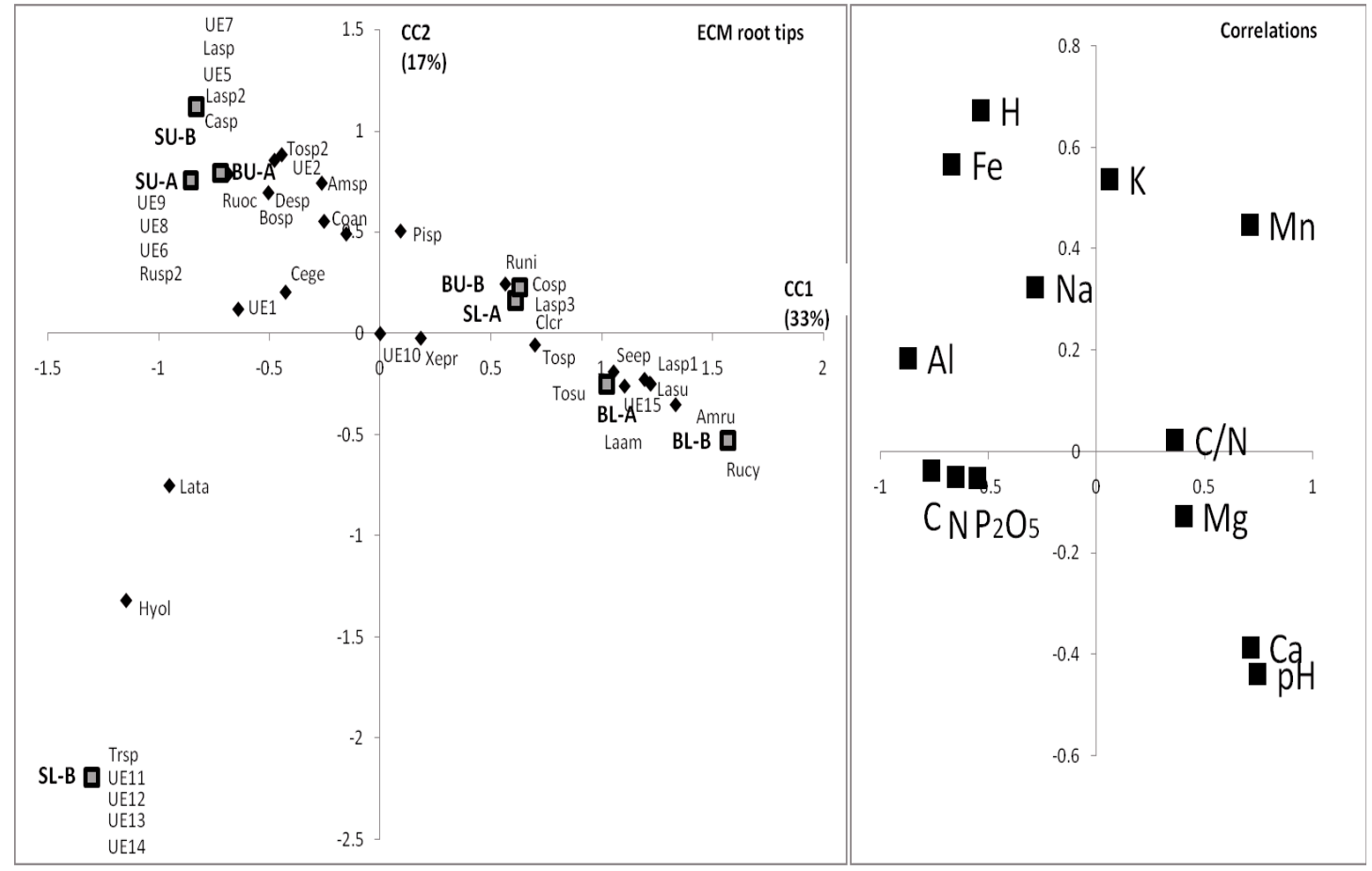

Figure 1. Canonical analysis based on the relative abundance of ECM root tips in each plot. Correlations: correlation circle between soil nutrient contents. ECM root tips: projection of the species and the 8 investigated plots on the plan of the two first canonical components. The first component (CC1) explained 33\% of the total variance, and the second one (CC2) 17\% (total: 50\%). S: spruce, B: Beech, U: untreated, L: limed, A: replicate A, B: replicate B. The name of each species is abbreviated according to Table I.

$\mathrm{P}_{2} \mathrm{O}_{5}$ contents (Fig. 1, Correlations). Predictions of canonical coordinates also showed the differentiation between limed and untreated plots following the first canonical component for beech and (ii) a differentiation between a limed plot (SL-A) and the other spruce ones (Fig. 1, ECM root tips). The correspondence analysis (CA) based on ECM root tip relative abundances revealed the opposition between species found in SL-A and both limed beech plots (Russula cyanoxantha, Tomentella sublilacina, Amanita rubescens, Clavulina cristata, Lactarius subdulcis) against those found in SL-B and all untreated plots (Cantharellus sp., UECM spp. 1 to 9, Russula ochroleuca, Cenococcum geophilum) (Fig. 1, ECM root tips). The second canonical component (17\% of the total variance, $y$ axis) was not strongly correlated with one environmental variable: it was to some extent correlated with available soil $\mathrm{Na}^{+}, \mathrm{K}^{+}$, $\mathrm{H}^{+}$and $\mathrm{Fe}^{3+}$ concentrations. The second canonical component also predicted an opposition between limed (especially SL-B) and untreated plots; the CA also showed a distinct group of 


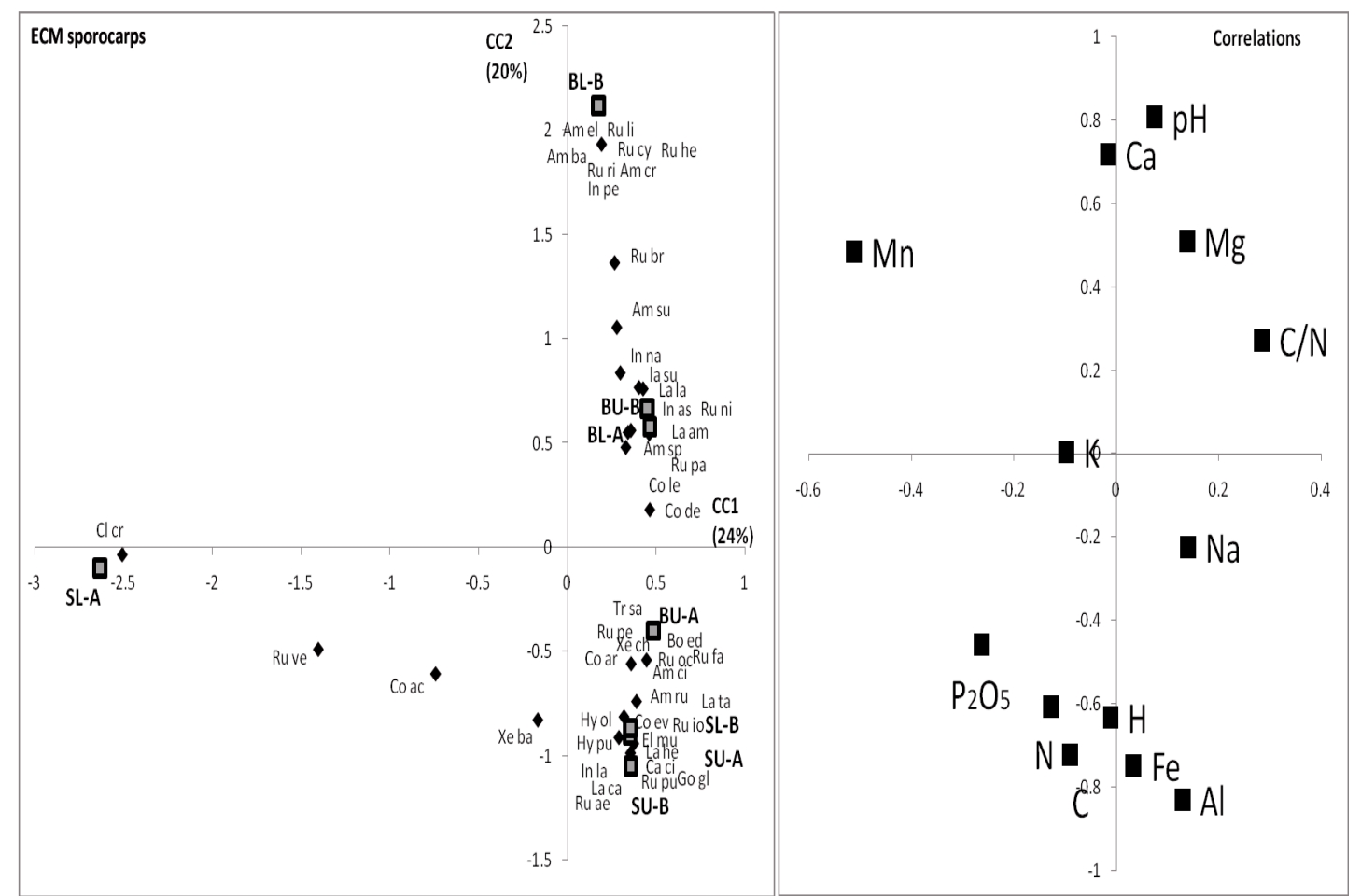

Figure 2. Canonical analysis based on the relative abundance of sporocarps of ECM species in each plot. Correlations: correlation circle between soil nutrient contents. ECM root tips: projection of the species and the 8 investigated plots on the plan of the two first canonical components. The first component (CC1) explained 24\% of the total variance, and the second one (CC2) 20\% (total: 44\%). S: spruce, B: Beech, U: untreated, L: limed, A: replicate A, B: replicate B. The name of each species is abbreviated according to Table I.

species close to the SL-B plot (Hygrophorus olivaceoalbus, Tricholoma sp., UECM spp. 11 to 14, Lactarius tabidus).

\subsection{Canonical analysis-Sporocarps of ECM fungi}

Results of a CCA based on relative abundances of ECM sporocarps clearly isolated SL-A and BL-A plots. The first canonical component (24\% of the total variance, $x$ axis) was positively correlated to concentrations in soil available $\mathrm{K}$ and negatively correlated with soil P concentration (Fig. 2, Correlations). Predictions of first canonical coordinate isolated clearly the SL-A plot from the other ones (Fig. 2, ECM sporocarps). The correspondence analysis (CA) based on sporocarp abundances also isolated species found in SL-A plot (particularly Clavulina cristata, and to a lesser extent Russula vesca and Cortinarius acutus). The second canonical component (20\% of the total variance, $y$ axis) strongly opposed $\mathrm{pH}$, exchangeable $\mathrm{Mn}, \mathrm{Ca}$ and $\mathrm{Mg}$ concentrations, to $\mathrm{Na}, \mathrm{Al}, \mathrm{H}, \mathrm{C}$ and $\mathrm{N}$ contents (Fig. 2, Correlations). Even if $\mathrm{BU}-\mathrm{B}$ was close to BL-A, predictions with the second canonical coordinate gradually isolated the beech limed plots from the untreated ones (Fig. 2, ECM sporocarps). The CA also distinguished species found only in the limed beech plots (Amanita battarae, Amanita crocea, Amanita eliae, Amanitopsis submembranacea, Inocybe petiginosa, Russula cyanoxantha, Russula heterophylla, Russula lilacea, Russula risigalina)
(Fig. 2, sporocarps). The species and plot ordination appeared as a horseshoe, showing that the sampled plots were probably distributed along an environmental gradient (Ramette, 2007).

\subsection{Canonical analysis-Sporocarps of saprophytic fungi}

The first canonical component (22\% of the total variance, $x$ axis) isolated the spruce plots from beech ones, and was correlated with $\mathrm{pH}, \mathrm{Ca}^{2+}$ and $\mathrm{Mn}^{2+}$, and negatively correlated with $\mathrm{Al}^{3+}$ (Fig. 3, Correlation). The second canonical component (19\% of the total variance, $y$ axis) was correlated with $\mathrm{K}$ and negatively correlated with soil P contents. Nevertheless, prediction of canonical components showed no distinction between limed and untreated plots. There was a clear block effect for spruce.

\section{DISCUSSION}

There was an inversion in the environmental variables associated with the two main canonical components between ECMs and fruiting bodies. Soil parameters relevant with the effect of liming (increased $\mathrm{Ca}^{2+}, \mathrm{Mg}^{2+}, \mathrm{pH}$, and decreased $\mathrm{Al}^{3+}$, total $\mathrm{N}$ and total C) were correlated with the first canonical component for ECMs and with the second one for fruiting 


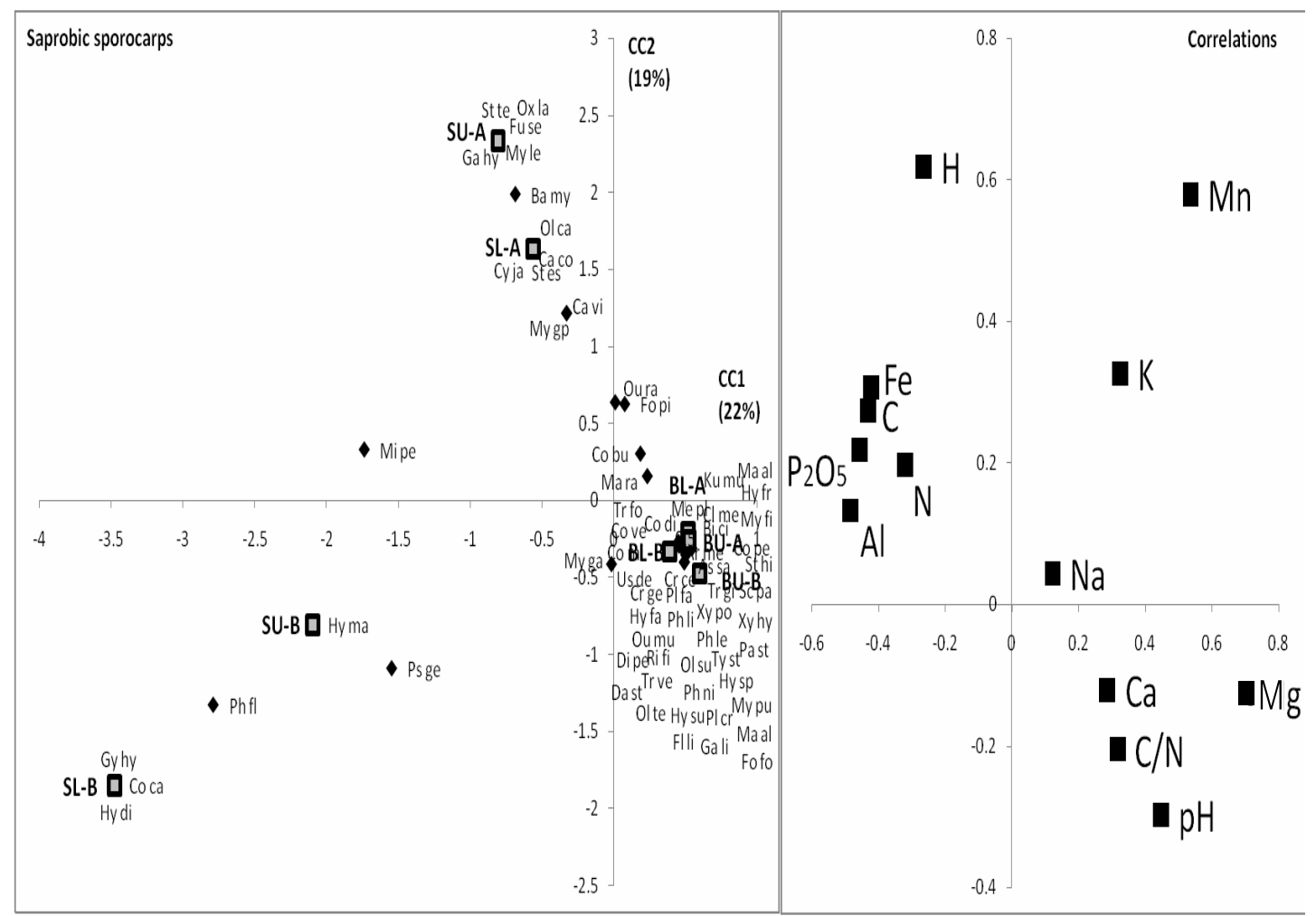

Figure 3. Canonical analysis based on the presence/absence of sporocarps of saprophytic species in each plot. Correlations: correlation circle between soil nutrient contents. ECM root tips: projection of the species and the 8 investigated plots on the plan of the two first canonical components. The first component (CC1) explained 22\% of the total variance, and the second one (CC2) 19\% (total: 41\%). S: spruce, B: Beech, U: untreated, L: limed, A: replicate A, B: replicate B. The name of each species is abbreviated with the first two letters of its genus name, followed by a space and the first two letters of its species name (see also supplementary Tab. II).

bodies. In contrast, increased exchangeable $\mathrm{K}^{+}$content and decreased in available $\mathrm{P}$ content were both correlated with the first canonical component for fruiting bodies and with the second one for ECMs, meaning that liming was a stronger factor of community structuring than tree host for ECMs, but not for sporocarps.

Concerning ECMs, there were many common species between spruce and beech plots. Among them, two ECM species were particularly repressed by liming: the most abundant ECM species in the overall community (Cenococcum geophilum), and another frequent ECM species (Russula ochroleuca). Cenococcum geophilum has often been reported as the dominant species of the ECM community (Baier et al., 2006; Courty et al., 2005; Dickie and Reich, 2005; Tedersoo et al., 2003). Generally a strong dominance of Cenococcum geophilum ECMs is reported in stressed forest stands, which is consistent with the status of moderate decline of the forest studied here. Acidophilic morphotypes as Russula ochroleuca were also repressed in limed plots. A similar decrease of abundance of this ECM in a liming experiment has already been reported (Qian et al., 1998). In contrast, liming also promoted relative abundance of the two ECM morphotypes Clavulina cristata and Tomentella sublilacina in both spruce and beech plots. These two species have often been found in surveys of ECM root tips and are considered as ubiquist (Buée et al., 2005; Frey et al., 2004; Köljalg et al., 2000; Taylor and Bruns, 1999; Tedersoo et al., 2006). Moreover, Tomentella sublilacina is an excellent competitor in mature forests (Taylor and Bruns, 1999). Liming also promoted relative abundance of the beech-specific and widespread ECM fungus Lactarius subdulcis (Courty et al., 2005). The main consequences of liming on ECM communities have therefore been the shift from Cenococcum geophilum and acidophilic species (i.e. and Russula ochroleuca) to a community dominated by ubiquist and highly competitive species. It is probable that the presence of these ubiquist species was due to the emergence of more nutrient-rich ecological niches in the limed areas, characterized by increased $\mathrm{pH}$ and contents of exchangeable $\mathrm{Ca}$ and $\mathrm{Mg}$, as observed on our sample site.

Community structuring of ECM fruiting bodies appeared more distinct between spruce and beech. Opposition between the SL-A plot and all the other ones was a prominent factor of community structuring. This plot was characterized by the very high abundance of Clavulina cristata and by a slight reduction of the abundance of Xerocomus badius, a mesoacidophilic fungus (Köttke et al., 1998). A possible "genet effect" (presence in one plot only because of a limited genet size) for Clavulina cristata is unlikely, because its sporocarps covered almost all the limed plot, and were nearly absent from the untreated one. 
The second factor of sporocarp community structuring gradually opposed beech limed and untreated plots; the limed plot coordinates were correlated with parameters characteristic of limed soils (exchangeable $\mathrm{Ca}$ and $\mathrm{Mg}, \mathrm{pH}$ ). Moreover, we observed that the 3 acidophilic species Amanita citrina, Cortinarius delibutus and Russula fageticola (in order of decreasing acidophilic optimum) were absent in the limed plots. The two latter ones were codominant species of the beech untreated plots, but none of the 3 species were found as ECM root tips. The relative abundance of Russula ochroleuca sporocarps also clearly decreased in the limed spruce plots, as observed by Agerer et al. (1998). As in the spruce limed plots, the abundance of an ubiquist fungus (Laccaria laccata) was also promoted by liming in beech. Nevertheless, acidophilic species as Russula brunneoviolacea also occurred in beech limed plots, showing that underlying soil acidity remained even after liming.

Some places in the overall limed area showed recolonization by Sphagnum species, showing a probable local soil re-acidification, often localized in hollows. It was the case in the SL-B plot, the fungal community of which was very close to that of the untreated plots. Moreover, we found in this plot very acidophilic species such as Lactarius helvus, in this plot. This means that the effect of liming on fungal communities is not uniform, probably because of the heterogeneous distribution of liming material in the soil, or by differences of soil drainage. The uneven distribution of some fungal species among the sampling plots (such as Clavulina cristata) may also be due to spatial soil heterogeneity in nutrient distribution, which is expected to significantly vary at a $5 \mathrm{~m}$ scale in forest soils (Gallardo, 2003). The SL-A plot, showing a strong dominance of Clavulina cristata in both ECM and sporocarp communities, presented high concentrations of exchangeable $\mathrm{Mn}$ : this might reflect preference of this ECM fungus to $\mathrm{Mn}$ rich soils. It is also likely that, knowing that ECM fungi vary a lot in niche size preference (Toljander et al., 2006), earthworm bioturbation after liming decreases the micro-scale soil heterogeneity, and thus destroys potential niches for fungi of less ecological amplitude. The bacterial communities, which are also affected by liming (Bäckman et al., 2003), could also indirectly elicit or repress some fungal species and thus influence fungal community structure.

The relative abundance of Russula ochroleuca sporocarps was affected by liming, as well as its fruiting behaviour. The sporocarps were widespread all over the 4 untreated plots, whereas they were always restricted to the base of trees in the limed ones, where most of the acidity occurs through stem flow. This suggests Russula ochroleuca as a good marker species of above and belowground effects of liming on ECM communities, using its ECM root tip relative abundance in several limed and untreated soils cores, and the position of its fruiting bodies in whole untreated and limed plots. This could be a quick and useful tool for foresters, because ECM root tips of Russula ochroleuca are frequent and easy to identify (pale yellow surface covered with bright yellow dots), as well as its sporocarps. Moreover, the spatial repartition of this species shows that local acidity can occur even in the limed plots and thus the soil spatial heterogeneity observed here can highly in- fluence fungal community structure. A similar liming-induced reduction of the abundance of acidophilic species at the profit of ubiquist, neutrophilic or competitive ones was observed on forest ground vegetation (Hallbäcken and Zhang, 1998) and mosses (Dulière et al., 2000). In the same way, high-dose liming decreased germination of acidophilic plant species (such as Vaccinium myrtillus) but increased germination of widespread ones (Primula veris, Senecio sylvaticus) (Olsson and Kellner, 2002). Nevertheless, liming did not have any significant effect on sporocarp and ECM community diversity (Shannon index) and equitability (Simpson index), even while the ECM diversity (Shannon index) had a tendency to decrease in limed plots. These results strongly tend to highlight the trivialization of all the communities after liming, due to the establishment of more nutrient-rich, and thus less selective, ecological niches.

Finally, among the environmental factors studied here, tree host was the most important for controlling the presence/absence of sporocarps of saprophytic fungal species. This has been mentioned in the literature for most of the lignin degrading basidiomycete species (Osono, 2007). Nevertheless, one non-specific species (Clitocybe nebularis) was present only in limed plots; other Clitocybe spp. and Collybia spp., known as unspecific to a tree host (Osono, 2007), were not affected by liming. The presence of many saprophytic fungi could be affected by interactions with other soil microbes (Osono, 2007), $\mathrm{C} / \mathrm{N}$ ratio (Lindahl et al., 2007), litter thickness (Yamashita and Hijii, 2006) or earthworm presence (Osono, 2007). Even if all these factors were more or less influenced by liming, we did not observe a specific pattern of saprophytic fungi community structure in the limed plots. We did not observe a significant reduction of fungal diversity (Shannon index and Simpson index) in plots where N concentration increased.

Some other soil factors that can affect sporocarp production were not studied here; they could have interfered with liming for structuring fungal communities. For example, soil humidity is a major factor of fungal fructification (Bonet et al., 2008; Kawakami et al., 2004), as well as soil temperature (Li, 1979), or $\mathrm{CO}_{2}$ concentration (Dahlberg and Van Etten, 1982). The age of the root system sampled can also be of critical importance for evaluating the ECM community structure (Gibson and Deacon, 1988). Finally, fungal species differ in their distribution: some occur in a very patchy way, whereas other ones can be solitary. This is true for sporocarps as well as for ECM root tips. The sampling strategy used here may overestimate the abundance of these patchy species compared to solitary ones. All these concerns, as well as the longer lifespan of a mycorrhiza (one week to one month) compared to that of a sporocarp (one day to one week), makes sporocarp survey more sensitive to climate than ECM community description. This can be a reason why the ECM communities studied here appear more sensitive to liming than sporocarp ones.

In conclusion, liming influenced very differently the structure of the communities of ECM root tips and sporocarps of symbiotic and saprophytic fungi. This forest practice had no obvious effect on saprophytic communities, but reduced the abundance of some acidophilic and meso acidophilic fruiting bodies of symbiotic fungi. This is compensated by the 
increased abundance of ubiquist species (Clavulina cristata and in a lesser extent Laccaria laccata). Finally, the same effect of liming (replacement of acidophilic and stress species by ubiquist and highly competitive ones) was observed in ECM root tip communities, and it was the main factor of community structuring, whatever the tree host. The appearance of ubiquist fungi was correlated with the development of new ecological niches, characterized by higher $\mathrm{pH}$ and exchangeable $\mathrm{Ca}^{2+}, \mathrm{Mg}^{2+}$ and $\mathrm{Mn}^{2+}$ concentrations. Nevertheless, the re-acidification of limed plots was observed in some depressed areas. These results show that, as for plants, liming leads to the trivialization of symbiotic fungal communities, even $15 \mathrm{y}$ after the treatment.

Acknowledgements: We thank Pr. C. Nys for providing the experimental site of Humont and for his expertise about liming, and the Office National des Forêts for allowing us to sample roots in the experimental plots. We also thank Dr. B. Marçais for useful comments on statistical analyses, and Dr P. Frey-Klett for very useful discussion about the results. The PhD scholarship of the first author was partly supported by the Lorraine Region. Part of this work has been funded by the Agence Nationale de la Recherche (contract FUNDIV, ANR-06-BDIV-006-01).

\section{REFERENCES}

Agerer R., 1987-1998. Colour atlas of ectomycorhizae, Einhorn-Verlag Eduard Dietenberger, Munich.

Agerer R., 2001. Exploration types of ectomycorrhizae: a proposal to classify ectomycorrhizal mycelial systems according to their patterns of differentiation and putative ecological importance. Mycorrhiza 11: $107-114$.

Agerer R., Taylor A.F.S., and Treu R., 1998. Effects of acid irrigation and liming on the production of fruit bodies by ectomycorrhizal fungi. Plant Soil 199: 83-89.

Bäckman J.S.K., Hermansson A., Tebbe C.C., and Lindgren P.E., 2003. Liming induces growth of a diverse flora of ammonia-oxidising bacteria in acid spruce forest soil as determined by SSCP and DGGE. Soil Biol. Biochem. 35: 1337-1347.

Baier R., Ingenhaag J., Blaschke H., Gottlein A., and Agerer R., 2006. Vertical distribution of an ectomycorrhizal community in upper soil horizons of a young Norway spruce (Picea abies [L.] Karst.) stand of the Bavarian Limestone Alps. Mycorrhiza 16: 197-206.

Bakker M.R., Garbaye J., and Nys C., 2000. Effect of liming on the ectomycorrhizal status of oak. For. Ecol. Manage. 126: 121-131.

Blaise T. and Garbaye J., 1983. Effets de la fertilisation minerale sur les ectomycorhizes d'une hetraie. Oecol. Plant. 18: 165-169.

Bonet J.A, Pukkala T., Fischer C.R., Palahí M., de Aragón J.M., and Colinas C., 2008. Empirical models for predicting the production of wild mushrooms in Scots pine (Pinus sylvestris L.) forests in the Central Pyrenees. Ann. For. Sci. 65: 206.

Buée M., Vairelles D., and Garbaye J., 2005. Year-round monitoring of diversity and potential metabolic activity of the ectomycorrhizal community in a beech (Fagus silvatica) forest subjected to two thinning regimes. Mycorrhiza 15: 235-245.

Courty P.E., Pritsch K., Schloter M., Hartmann A., and Garbaye J. 2005. Activity profiling of ectomycorrhiza communities in two forest soils using multiple enzymatic tests. New Phytol. 167: 309-319.

Dahlberg A., 2001. Community ecology of ectomycorrhizal fungi: an advancing interdisciplinary field. New Phytol. 150: 555-562.
Dahlberg K.R. and van Etten J.L., 1982. Physiology and biochemistry of fungal sporulation. Ann. Rev. Phytopathol. 20: 281-301.

Dickie I.A. and Reich P.B., 2005. Ectomycorrhizal fungal communities at forest edges. J. Ecol. 93: 244-245.

Duchaufour Ph. and Bonneau M.,1959. Une méthode nouvelle de dosage du phosphore assimilable dans les sols forestiers. Bull. AFES 4: 193198.

Dulière J.F., De Bruyn R., and Malaisse F., 2000. Changes in the moss layer after liming in a Norway spruce (Picea abies [L.] Karst.) stand of Eastern Belgium. For. Ecol. Manage. 136: 97-105.

Frank J. and Stuanes A. O., 2003. Short-term effects of liming and vitality fertilization on forest soil and nutrient leaching in a scots pine ecosystem in Norway. For. Ecol. Manage. 176: 371-386.

Frey S.D., Knorr M., Parrent J.L., and Simpson R.T., 2004. Chronic nitrogen enrichment affects the structure and function of the soil microbial community in temperate hardwood and pine forests. For. Ecol. Manage. 196: 159-171.

Gallardo A., 2003. Spatial variability of soil properties in a floodplain forest in northwest spain. Ecosystems 6: 564-576.

Garbaye J., 1990. Pourquoi et comment observer l'état mycorhizien des plants forestiers. Rev. For. Fr. XLII: 35-47

Garbaye J., Kabre A., Le Tacon F., Mousain D., and Piou D., 1979. Fertilisation minérale et fructification des champignons supérieurs en hêtraie. Ann. Sci. For. 36: 151-164.

Gardes M. and Bruns T.D., 1993. ITS primers which enhanced specificity fot basidiomycetes-application to the identification of mycorrhizae and rusts. Mol. Ecol. 2: 113-118.

Gibson F. and Deacon J.W., 1988. Experimental study of establishment of ectomycorrhizas in different regions of birch root systems. Trans. Br. Mycol. Soc. 91: 239-251.

Hallbäcken L. and Zhang L. Q., 1998. Effects of experimental acidification, nitrogen addition and liming on ground vegetation in a mature stand of Norway spruce (Picea abies [L.]) Karst.) in SE Sweden. For. Ecol. Manage. 108: 201-213.

Haug I. and Feger K.H., 1990. Effects of fertilization with MgSO4 and (NH4)2SO4 on soil solution chemistry, mycorrhiza and nutrient content of fine roots in a Norway spruce stand. Water Air Soil Pollut. 54: 453-467.

Ihaka R. and Gentleman R., 1996. R: a language for data analysis and graphics. J. Comput. Graph. Stat. 5: 299-314

Kawakami A., Matsumoto N., and Naito S., 2004. Enviroonmental factors influencing sporocarp formation in Typhula ishikariensis. J. Gen. Plant. Pathol. 70: 1-6.

Köljalg U., Dahlberg A., Taylor A.F.S., Larsson E., Hallenberg N., Stenlid J., Larsson K.H., Fransson P.M., Karen O., and Jonsson L., 2000. Diversity and abundance of resupinate thelephoroid fungi as ectomycorrhizal symbionts in Swedish boreal forests. Mol. Ecol. 9: 1985-1996.

Kottke I., Qian X.M, Pritsch K., Haug I., and Oberwinkler F., 1998. Xerocomus badius-Picea abies, an ectomycorrhiza of high activity and element storage capacity in acidic soil. Mycorrhiza 7: 267-275.

Kreutzer K., 1995. Effects of forest liming on soil processes. Plant Soil 168: 447-470.

Li C.Y., 1979. Light and temperature induced sporocarp formation of Phellinus weirii. Can. J. For. Res. 9: 535-538.

Lindahl B., Ihrmark K., Boberg J., Trumbore S.E., Högberg P., Stenlid J., and Finlay R.D., 2007. Spatial separation of litter decomposition and mycorrhizal nitrogen uptake in a boreal forest. New Phytol 173: 611-620. 
Nowotny I., Dähne J., Klingelhöfer D., and Rothe G.M., 1998. Effect of artificial soil acidification and liming on growth and nutrient status of mycorrhizal roots of Norway spruce (Picea abies [L.]) Karst.). Plant Soil 199: 29-40.

Olsson B.A. and Kellner O., 2002. Effects of soil acidification and liming on ground flora establishment after clear-felling of Norway spruce in Sweden. For. Ecol. Manage. 158: 127-139.

Osono T., 2007. Ecology of ligninolytic fungi associated with leaf litter decomposition. Ecol. Res. 22: 955-974.

Persson T., 1988. Effects of liming on soil fauna in forests. A literature review. Swed. Environ. Prot. Board Report. Rep. 3418.

Qian X.M., Köttke I., and Oberwinkler F., 1998. Influence of liming and acidification on the activity of the mycorrhizal communities in a Picea abies (L.) Karst. Stand. Plant Soil 199: 99-109.

Ramette A., 2007. Multivariate analyses in microbial ecology. FEMS Microbiol. Ecol. 62: 142-160

Renaud J.P., Picard J.F., Richter C., and Nys C., 2000. Restauration de sols forestiers acides par un amendement calco-magnesien. Cas du Massif Vosgien et de l'Ardenne. Rapport DERF-ONF-INRA, 39 p.
Taylor D.L. and Bruns T.D, 1999. Community structure of ectomycorrhizal fungi in a Pinus muricata forest: minimal overlap between the mature forest and resistant propagule communities. Mol. Ecol. 8: $1837-1850$

Tedersoo L., Köljalg U., Hallenberg N., and Larsson K.H., 2003. Fie scale distribution of ectomycorrhizal fungi and roots across substrate layers including coarse woody debris in a mixed forest. New Phytol. 159: $153-165$.

Tedersoo L., Suva T., Larsson E., and Köljalg U., 2006. Diversity and community structure of ectomycorrhizal fungi in a wooded meadow. Mycol. Res. 110: 734-748.

Thomas, G.W., 1982. Exchangeable cations. In: Methods of soil Analysis. Page A.L. et al. (Eds.), Agronomy 9: 154-157.

Toljander J.F., Eberhardt U., Toljander Y.K., Paul L.R., and Taylor A.F.S., 2006. Species composition of an ectomycorrhizal fungal community along a local nutrient gradient in a boreal forest. New Phytol. 170: 873-884.

Ulrich B., 1983. Effects of acid deposition. Acid deposition, pp.31-41. 